\title{
Dynamics of a network fluid within the liquid-gas coexistence region
}

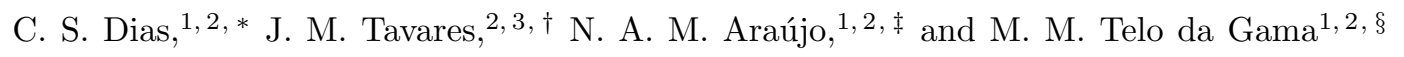 \\ ${ }^{1}$ Departamento de Fúsica, Faculdade de Ciências, \\ Universidade de Lisboa, 1749-016 Lisboa, Portugal \\ ${ }^{2}$ Centro de Física Teórica e Computacional, Universidade de Lisboa, 1749-016 Lisboa, Portugal \\ ${ }^{3}$ Instituto Superior de Engenharia de Lisboa, ISEL, \\ Avenida Conselheiro Emídio Navarro, 1 1950-062 Lisboa, Portugal
}

\begin{abstract}
Low-density networks of molecules or colloids are formed at low temperatures when the interparticle interactions are valence limited. Prototypical examples are networks of patchy particles, where the limited valence results from highly directional pairwise interactions. We combine extensive Langevin simulations and Wertheim's theory of association to study these networks. We find a scale-free (relaxation) dynamics within the liquid-gas coexistence region, which differs from that usually observed for isotropic particles. While for isotropic particles the relaxation dynamics is driven by surface tension (coarsening), when the valence is limited, the slow relaxation proceeds through the formation of an intermediate non-equilibrium gel via a geometrical percolation transition in the Random Percolation universality class.
\end{abstract}

The formation of stable low-density structures of interconnected molecules or colloids is an open question that attracted researchers in the last decade [1 5. These unique structures are simultaneously of low packing fraction and resilient to mechanical perturbations. The former can be achieved through limited valence of the individual constituents. The latter requires sufficiently strong interparticle bonding. However, establishing strong bonds leads to rough energy landscapes, challenging the formation of the final equilibrium structures [6, 7]. Kinetically arrested structures are usually obtained, which may be significantly different from the thermodynamic ones.

Colloidal valence may be engineered by chemically patterning the colloidal surfaces with attractive patches 8 18. Studies of equilibrium phase diagrams have shown that at low valence, due to the decrease in the density of the liquid binodal, a gel-like low density structure is thermodynamically stable (equilibrium) for a wide range of attractive strengths and densities 19 . However, inside the coexistence region, the system is expected to phase separate into colloid-rich and colloidal-poor phases 20 25. Nevertheless, as the lifetime of thermal reversible bonds increases exponentially with the attraction, an arrested gel is formed [26] in line with experimental observations for mixtures of colloids and polymers 27 . These non-ergodic gels are significantly different from the thermodynamic ones.

In what follows, we investigate the collective dynamics of limited-valence particles in various regions of the phase diagram. We show that the interplay between relaxation mechanisms at different length and time scales leads to distinct dynamics at low and high temperatures. At high temperatures the dynamics is driven by sequences of bond breaking and forming events and it is characterized by an Arrhenius relaxation time related to the thermal reversibility of the bonds. At low temperatures, however, a scale-free dynamics is observed.

\section{RESULTS}

We consider a three-dimensional system of spherical particles of diameter $\sigma$ with three patches along the equator with an opening angle of $2 \pi / 3$. The core-core interaction is pairwise repulsive, described by a Yukawa-like potential $V_{Y}=\frac{A}{\alpha} \exp (-\alpha[r-\sigma])$, where $A$ is the strength of the potential, $r$ is the distance between the particles centers, and $\alpha^{-1}$ is the range of the interaction. The short-range patch-patch attraction is described by an inverse Gaussian potential, $V_{G}=-\varepsilon \exp \left(-r_{p}^{2} / \xi^{2}\right)$, where $\varepsilon$ is the strength of the attraction, $\xi=0.1 \sigma$ the width of the Gaussian, and $r_{p}$ is the patch-patch distance. For simplicity, patch-particle interactions are neglected. In order to follow the collective dynamics, we integrate the corresponding Langevin equations for the translational and rotational motions (see Methods for further details).

Figure 1(a) shows the temperature dependence of the bonding fraction, $p_{b}$, defined as the fraction of patches that are bonded. The squares are obtained by extrapolating the time-dependence of $p_{b}$ to its asymptotic value from the numerical data for three different system sizes, up to $L=32$, which is constant within the error bars (see Supplementary Fig. S2). The solid (red) line corresponds to the equilibrium value as predicted by Wertheim's firstorder perturbation theory (details in the Methods section). While at high temperatures the numerical equilibrium results overlap, at low temperatures the bonding fraction is systematically lower than the equilibrium value and does not converge to unity at zero temperature. This signals the presence of an arrested gel in the coexistence region (see snapshots in Fig. 1(a) and the equilibrium phase diagram in Fig. 1(b)). Note that the numerical data are the asymptotic values, suggesting that the thermodynamic structures are not accessible.

Far-from equilibrium the asymptotic structures depend on the dynamics. We have considered two limiting initial configurations with the same number of particles: 

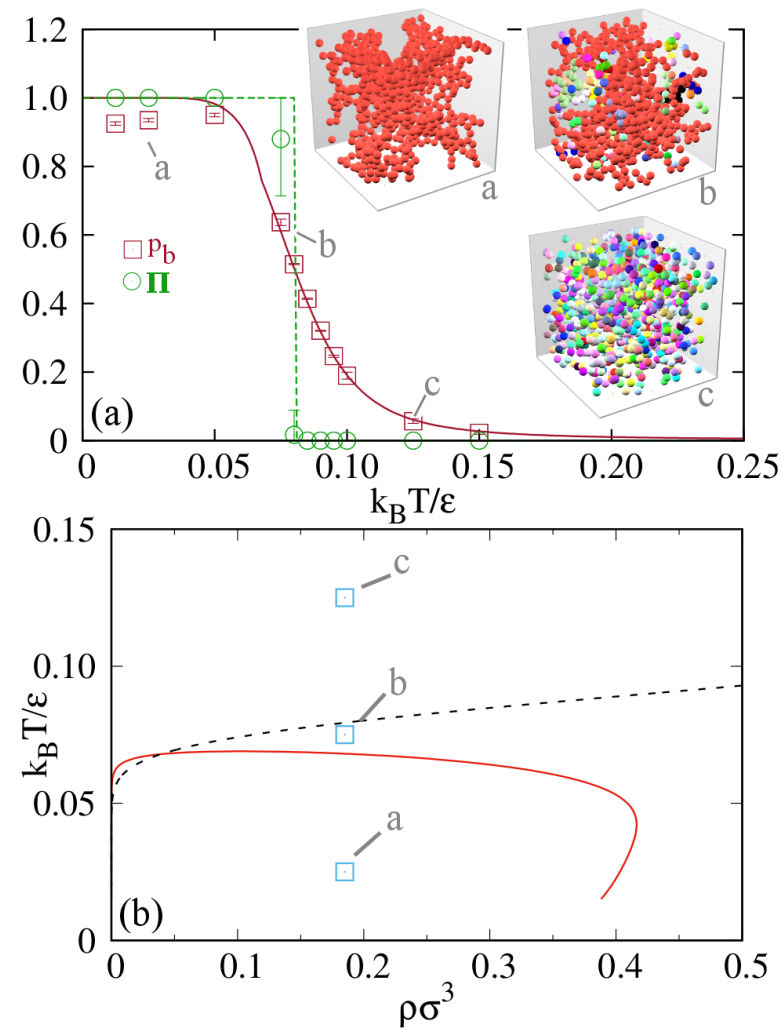

FIG. 1. (a) The (red) squares are the bonding fraction $p_{b}$ as a function of the rescaled temperature $k_{B} T / \varepsilon$, obtained by extrapolating the time-dependence of $p_{b}$ to its asymptotic value from the numerical data for systems of linear size $L=\{8,16,32\}$. The solid line is the equilibrium value predicted by Wertheim's first-order perturbation theory of association (see Methods for further details). The (green) circles are the wrapping probability $\Pi$, defined numerically as the fraction of samples with an aggregate of bonded particles that touches simultaneously the top and the bottom boundaries of the simulation box. The dashed line corresponds to the mean-field prediction for $\Pi$, which is a step function at $k_{B} T^{*} / \varepsilon$, where $p_{b}\left(k_{B} T^{*} / \varepsilon\right)=1 / 2$. Simulations were performed at a density $0.2 \sigma^{-3}$. (b) Density-temperature phase diagram, where the density $\rho=N / V$ is the number of particles per unit volume and the temperature is the rescaled temperature $k_{B} T / \varepsilon$. The solid (red) line is the coexistence line and the dashed (black) line is the percolation line. Insets are snapshots at $k_{B} T / \varepsilon$ f a) 0.025 , b) 0.075 , and c) 0.125 , where different colors correspond to different aggregates of bonded particles. Numerical results were obtained by averaging over 10 samples, for a box of linear size $L=16$, in units of the particle diameter, and $N=800$ particles.

A tree-like gel (single aggregate) and a fully unbonded suspension. The former was grown by ballistic aggregation and loopless irreversible bond formation [28] and corresponds to relaxing a structure grown at very low temperature 29. The latter is equivalent to quenching a colloidal solution from a much higher temperature. Figure 2(a) shows the time evolution of the fraction of
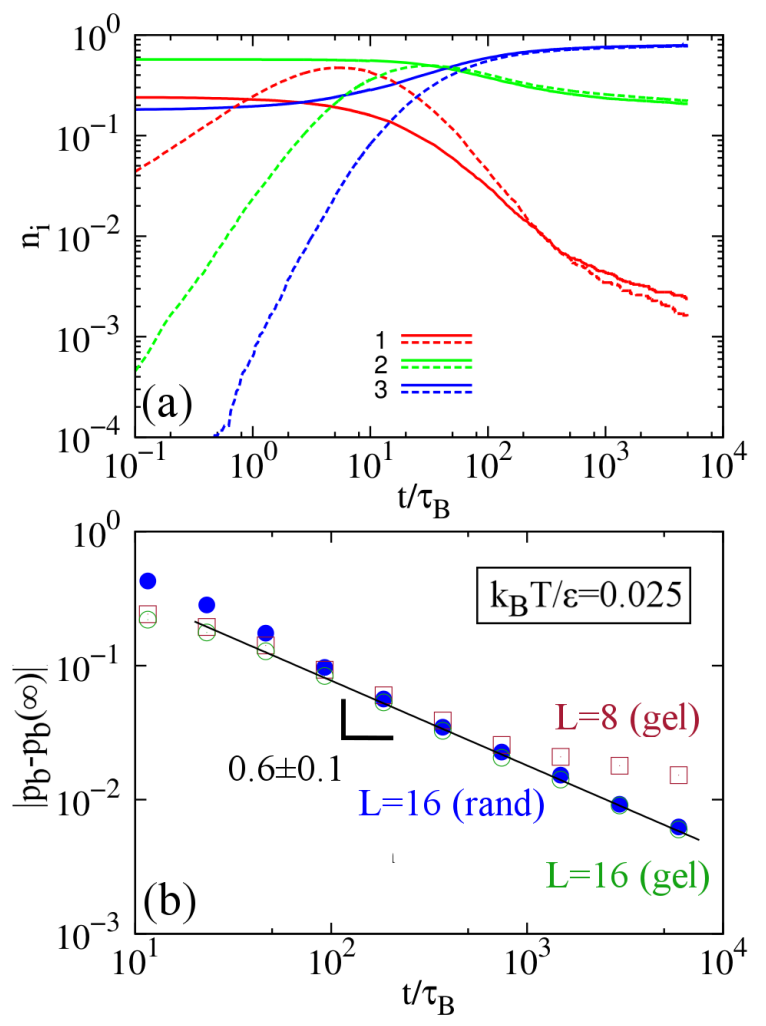

FIG. 2. (a) Time evolution of the fraction of particles with one (red), two (green), and three (blue) bonds, at $k_{B} T / \varepsilon=0.025$ (below the coexistence curve). The solid lines are for the relaxation of a tree-like gel grown by ballistic aggregation and loopless irreversible bond formation. The dashed lines are for the aggregation of an initial fully unbonded suspension of patchy particles. Time is rescaled by the Brownian time $\tau_{B}=\sigma^{2} / D_{t}$, where $\sigma$ is the particle diameter and $D_{t}$ is the self-diffusion coefficient of a single particle at short times. Results are averages over 100 samples for $t / \tau_{B}<10$ and and 20 samples for $t / \tau_{B}>10$, of a cubic box of length $L=16$, in units of the particle diameter. (b) Convergence of the bonding fraction to the asymptotic value $\left|p_{b}-p_{b}(\infty)\right|$ at low temperature $\left(k_{B} T / \varepsilon<0.075\right)$, for initial conditions of tree-like gels grown by ballistic aggregation (gel) and fully unbonded suspensions (rand), where the time is rescaled by the Brownian time $\tau_{B}=\sigma^{2} / D_{t}$, obtained by averaging over 20 samples in a box of length $L=\{8,16\}$ (same density), in units of the particle diameter. Simulations were performed at a density $0.2 \sigma^{-3}$.

particles with one $\left(n_{1}\right)$, two $\left(n_{2}\right)$, and three bonds $\left(n_{3}\right)$ for both initial configurations at $k_{B} T / \varepsilon=0.025$. When starting from a tree-like gel, $n_{3}$ increases monotonically, while $n_{1}$ and $n_{2}$ decrease, suggesting that structural relaxation is driven by maximizing the bonded patches. When starting from a completely unbonded system, the kinetic pathway is significantly different, resembling a typical aggregation process. $n_{1}$ and $n_{2}$ initially increase due to the formation of small, chain-like, aggregates but eventually peaks at an intermediate time, when branch- 


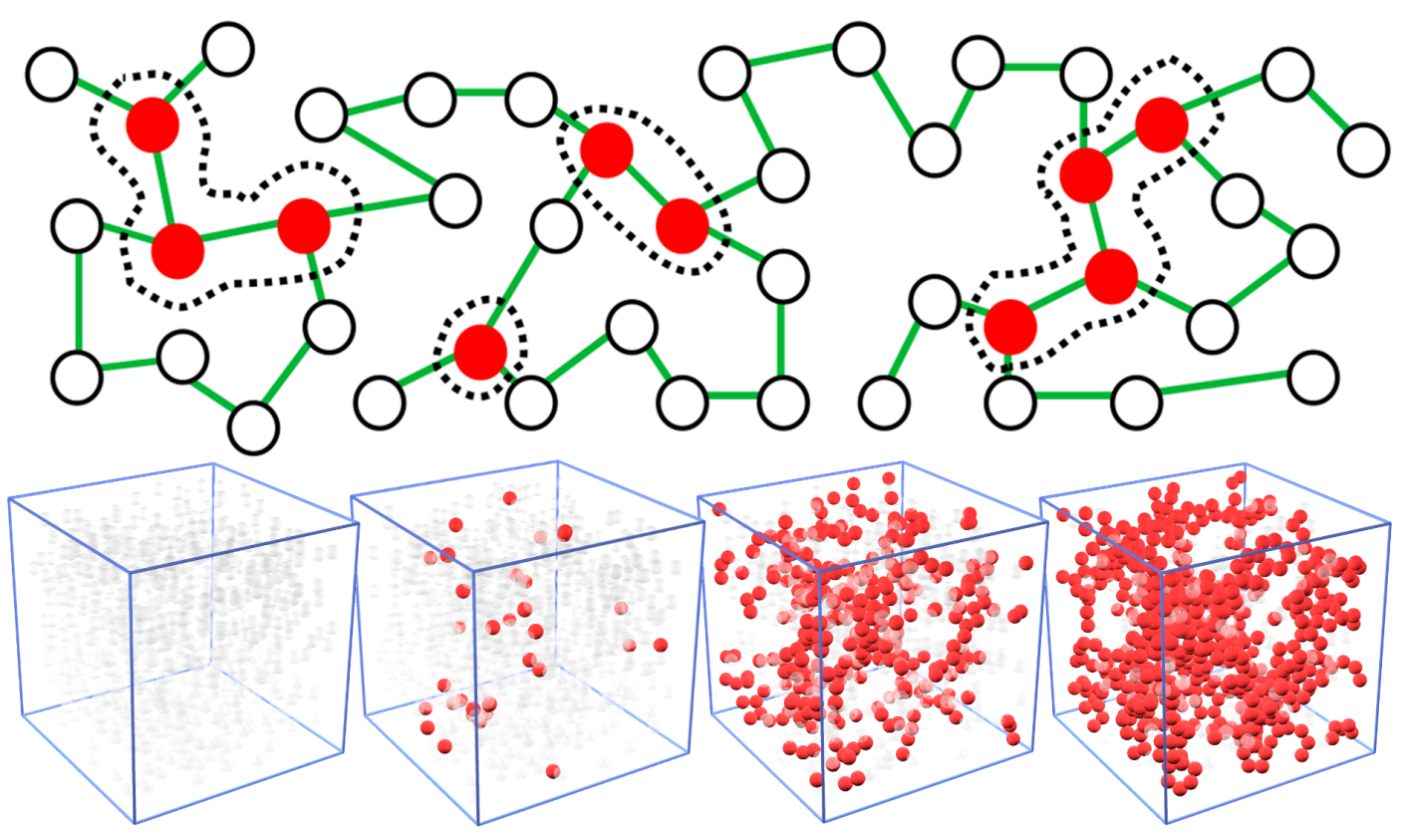

FIG. 3. Relaxation mechanisms responsible for the scale-free dynamics within the gas-liquid phase coexistence. (top) Schematic representation of the relaxation mechanism for network fluids mediated by concerted rearrangements of interconnected particles. Red circles represent the liquid phase (three bonds) and black circles represent the gas phase. Green lines are the network fluid bonds. (bottom) Langevin Dynamics of a network fluid quenched from a high temperature to a low temperature into the coexistence region. The time increases from left to right for a system of linear size $L=16$. Red particles represent the liquid phase.

ing and loop closing dominate. Note that, for the two drastically different initial configurations and pathways, the distributions of particles with one, two, and three bonds, are surprisingly similar, sufficiently long times, albeit different from the thermodynamic (equilibrium) ones (fully bonded, $n_{3}=1$ ). This result suggests the existence of high energy barriers to relax to the equilibrium configuration and reveals that the geometrical kinetic structures are statistically robust.

To characterize the dynamics, in the low temperature limit, we follow the convergence of $p_{b}$ to its asymptotic value $p_{b}(\infty)$ (see Fig. 2(b)). In Fig.2(b), we observe the size dependence of $p_{b}(\infty)$ for systems ranging from $L=8$ to $L=16$. In this regime, within the coexistence region (see Fig. 1(b)), $p_{b}$ follows a power law in time, with an exponent of $0.6 \pm 0.1$. The finite-size study shows that the power law regime increases with the size of the system. The numerical data is consistent with the same exponent for different initial conditions (tree-like gel and fully unbonded) and temperatures in the coexistence region (see Supplementary Fig. S1).

Previous studies for isotropic particles suggest that, within the gas-liquid coexistence region, the relaxation dynamics is driven by the formation of individual clusters that coarsen to minimize the surface tension 30 . By contrast here, due to the strong particle-particle interaction, a single cluster spanning the entire system is formed well before the scale-free behavior is observed (see Supplementary Fig. S3). This is similar to what has been observed previously in other network fluids, namely Laponite [31. There, the heterogeneity of the observed cluster suggests a coarsening process, where highly bonded dense zones of the aggregate represent the liquid phase. In order to discard that possibility, we assume that only particles with three bonds are in the liquid phase and identify the clusters of bonded particles. We proceed to analyze their relaxation dynamics (see a schematic representation in Fig. 3p. Since all particles belong to the gel-like network the growth of the liquid phase evolves through the formation of new bonds of particles with one or two bonds. Snapshots of the system at the bottom of Fig. 3 illustrate this behavior. For surface tension driven relaxation (coarsening) the characteristic size of the clusters $l^{*}$ should scale as $l^{*} \sim t^{1 / 3}$. However, we observe a clearly different exponent, as $\left\langle s>\sim t^{0.3 \pm 0.1}\right.$ implies $l^{*} \sim t^{0.10 \pm 0.04}$ (considering $l \sim\left\langle s>^{1 / 3}\right.$ ), where $\langle s\rangle$ is the mean cluster size, and suggests a different mechanism (see Supplementary Fig. S3 for more details). We should note that the surface tension of network fluids is ultra-low [32] and this is likely to render coarsening in these systems rather ineffective.

In Fig. 4 we report the analysis of the kinetics of the clusters of liquid particles. Figure 4(a) shows the time dependence of the fraction of particles in the largest clus- 


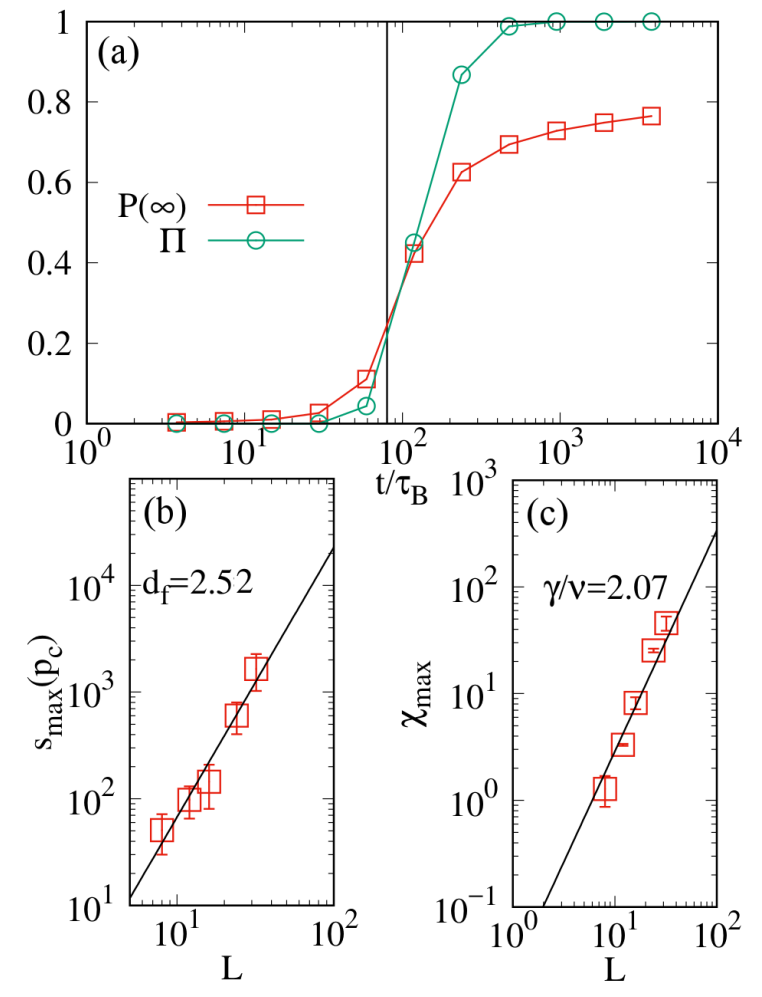

FIG. 4. (a) The fraction of particles in the largest cluster $P(\infty)$ and wrapping probability $\Pi$ of the aggregate of liquid particles (with three-bonds) as a function of time for a system of linear size $L=16$, at $k_{B} T / \varepsilon=0.025$. Time is rescaled by the Brownian time $\tau_{B}=\sigma^{2} / D_{t}$, where $\sigma$ is the particle diameter and $D_{t}$ its diffusion coefficient. (b) Size of the largest cluster at the percolation threshold, represented by a vertical solid line in (a), for system sizes $L=\{8,12,16,24,32\}$. The solid line represents the theoretical slope for random percolation where $s_{\max } \sim L^{d_{f}}$ with $d_{f}=2.52$ the largest aggregate fractal dimension at threshold. (c) Order parameter variance $\chi$, at the percolation threshold, for system sizes $L=\{8,12,16,24,32\}$. The solid line represents the theoretical slope for random percolation where $\chi_{\max } \sim L^{\gamma / \nu}$ with $\gamma / \nu=2.07$. All simulations were performed at a density $0.2 \sigma^{-3}$.

ter and the wrapping probability, defined as the probability of finding a cluster that touches opposite sides of the box in the vertical direction. We find a percolation transition at $t / \tau_{\mathrm{B}} \approx 10^{2}$. Figures $4(\mathrm{~b})$ and $(\mathrm{c})$ show the system-size dependence of the number of particles in the largest cluster and its variance at the percolation threshold. The results are consistent with power-law scaling with the exponents of the Random Percolation universality class [33, 34, suggesting that the relaxation dynamics of the liquid clusters goes through a percolation transition. This could explain why the late time relaxation dynamics of the liquid is independent of the initial condition.

In summary, we investigated the relaxation dynamics of particles with limited valence, and found a new dynamical regime within the coexistence region. The slow thermal reversibility of the bonds induces structural disorder that is quenched on the experimental timescale effectively hindering the relaxation of the structure towards thermodynamic equilibrium. The numerical data for gel aging within the coexistence region is consistent with a universal exponent, $0.6 \pm 0.1(\approx 2 / 3)$, for the evolution of the bonding fraction, which is significantly lower than what is known for surface tension driven coarsening.

\section{DISCUSSION}

Our study reveals a new relaxation mechanism of the gel network, where the liquid does not coarsen by interfacial fluctuations as a result of very long lived bonds (practically irreversible) and ultra-low interfacial tension. The relaxation mechanism proceeds through the relaxation of the network and not through local rearrangements of a small number of neighboring particles. The liquid phase does not grow by the addition of clusters of nearby particles but through geometrical relaxation events occurring over the whole network. At least initially these geometrical relaxation events are random. The power-law relaxation is also a hint of these collective rearrangements.

Previous studies for Lennard-Jones particles, within the solid-gas coexistence, revealed a power law time dependence for the size of the largest cluster at very low temperatures, distinct from (surface tension driven) coarsening 35. Their numerical data suggests that the exponent decreases with the temperature. Although particle diffusion is also strongly suppressed by the gel-like structure, our numerical data is consistent with a robust exponent over the range of temperatures considered. A crucial difference from the Lennard-Jones fluid is that in the network fluid the liquid clusters are also immobile.

Finally, a comment on other low-valence systems is in order. Numerical studies of network fluids of particles with (two) oppositely oriented dipole moments also suggest a power-law decay of the bond correlation function 36, 37. These results, obtained by Monte Carlo (equilibrium) simulations hint at a dynamic slowing down driven by gel formation. It would be interesting to investigate the dynamics of these dipolar systems and to compare their aging to that reported here. There is also the question of the dependence of the dynamics on the particles valence. At low valence, crowding effects are absent. However, the network density increases with valence [3841. and as the valence increases, crowding effects are expected to play a role. Khalil et al. investigated structural arrest transitions in polymer suspensions with cross linkers 42, a problem that may be related to mixtures of limited valence particles. An interplay between the glass and the gel transitions was observed depending on the polymer and linker concentrations. Future work could 
address similar questions in network fluids and/or their mixtures in order to elucidate the role of the valence.

\section{METHODS}

Model and simulations. We consider spherical particles, all of the same size, with three identical patches distributed along the equator. The core-core interaction is repulsive, described by a Yukawa-like potential,

$$
V_{Y}(r)=\frac{A}{\alpha} \exp (-\alpha[r-\sigma]),
$$

where $\sigma$ is the diameter of the particles, $A /\left(\alpha k_{B} T\right)=$ 0.25 is the interaction strength and $\alpha / \sigma=0.4$ the inverse screening length. The core-core interaction is truncated at a cutoff distance $r_{c}=1.5 \sigma$ (at $r=r_{c}$ the potential is $\left.10^{-9} A / \sigma\right)$. The patch-patch interaction is described by an attractive inverted Gaussian potential [43, 44,

$$
V_{G}\left(r_{p}\right)=-\varepsilon \exp \left(-r_{p}^{2} / \xi^{2}\right)
$$

where $\varepsilon$ is the strength of the attraction, $\xi=0.1 \sigma$ the width of the Gaussian, and $r_{p}$ the patch-patch distance. This interaction is truncated also at a cutoff distance $r_{p c}=\sigma$. For this set of parameters, we expect at most one bond per patch.

To resolve the stochastic trajectories of the particles, we integrate the Langevin equations of motion for the translational degrees of freedom,

$$
m \dot{\vec{v}}(t)=-\nabla_{\vec{r}} V(\vec{r})-\frac{m}{\tau_{t}} \vec{v}(t)+\sqrt{\frac{2 m k_{B} T}{\tau_{t}}} \vec{\xi}(t),
$$

and the rotational ones,

$$
I \dot{\vec{\omega}}(t)=-\nabla_{\vec{\theta}} V(\vec{\theta})-\frac{I}{\tau_{r}} \vec{\omega}(t)+\sqrt{\frac{2 I k_{B} T}{\tau_{r}}} \vec{\xi}(t) .
$$

We use the velocity Verlet scheme with a time step $\Delta t=1 \times 10^{-5}$, in units of the Brownian time (time to diffuse one particle diameter), and the Largescale Atomic/Molecular Massively Parallel Simulator (LAMMPS) for efficient simulations 45. $\vec{v}$ and $\vec{\omega}$ are the translational and angular velocities, $m$ and $I$ are the mass and moment of inertia of the particles, $V$ is the pairwise potential, and $\vec{\xi}(t)$ is the stochastic term drawn from a random distribution with zero mean. We consider the damping time for the translational motion,

$$
\tau_{t}=\frac{m}{6 \pi \eta R}
$$

From the Stokes-Einstein-Debye relation [46],

$$
\frac{D_{r}}{D_{t}}=\frac{3}{4 R^{2}}
$$

and thus the rotational damping time is $\tau_{r}=10 \tau_{t} / 3$.

Wertheim's theory. The thermodynamic equilibrium properties of the model were calculated using Wertheim's first order perturbation theory (see, e.g. 47]). The Helmholtz free energy per particle $f$ is the sum of a reference free energy $f_{\text {ref }}$ and a perturbation (arising from bonding) $f_{b}, \beta f=\beta f_{\text {ref }}+\beta f_{b}$ where $\beta=1 / k_{B} T$. The reference potential was taken to be the repulsive Yukawa, $V_{Y}(r)$, and the perturbation (that promotes bonding) the attractive patch-patch interaction, $V_{G}\left(r_{p}\right)$. The bonding term is given by,

$$
\beta f_{b}=n \ln \left(1-p_{b}\right)-\frac{n}{2}\left(1-p_{b}\right),
$$

where $n$ is the number of (identical) patches $(n=3)$ and $p_{b}$ is the bonding fraction, which in the thermodynamic limit corresponds to the fraction of bonded patches. We approximate $f_{\text {ref }}$ by the hard sphere free energy (in the Carnahan-Starling approximation) of a system of particles with an effective diameter $D$ calculated within the Barker-Henderson approximation [48,

$$
D=\int_{0}^{+\infty}\left(1-\exp \left[-\beta V_{Y}(r)\right]\right) d r
$$

The probability $p_{b}$ was calculated using the law of mass action,

$$
p_{b}=n \rho \Delta\left(1-p_{b}\right)^{2}
$$

where $\rho$ is the number density defined as the number of particles per unit volume. $\Delta$ is the integral of the Mayer function of the patch-patch interaction,

$$
\Delta=\frac{1}{(4 \pi)^{2}} \int d \vec{r} \int d \hat{r}_{1} \int d \hat{r}_{2}\left[\exp \left(-\beta V_{G}\left(r_{p}\right)\right]-1\right) g_{r e f}(r)
$$

where $\vec{r}$ is the vector between the centers of particles 1 and 2 , and $\hat{r}_{i}$ is the unit vector that defines the position of a patch on particle $i$ relative to the center of that particle. $g_{\text {ref }}(r)$ is the pair correlation function of the reference system, which is approximated by the contact value of the pair correlation function of a system of hard spheres of diameter $D: g_{\text {ref }}(r)=g_{H S}(r=D)=(1-\eta / 2) /(1-\eta)^{3}$, with $\eta=\pi / 6 D^{3} \rho$. The multiple integral 10 is then reduced to a sum of simple integrals through the following steps (a simple extension of Ref. [49]):

1 Define $z=r_{p} \equiv\left|\vec{r}+\sigma / 2\left(\hat{r}_{2}-\hat{r}_{1}\right)\right|$ and $x=\mid \vec{r}+$ $\sigma / 2 \hat{r}_{2} \mid$.

2 Simplify 10 using the change of variables: (i) from $\theta_{1}$ to $z^{2}$, in the integration over $\hat{r}_{1}$, with $z^{2}=x^{2}+\sigma^{2} / 4-x \sigma \cos \theta_{1}$; (ii) from $\theta_{2}$ to $x^{2}$, in the integration over $\hat{r}_{2}$, with $x^{2}=r^{2}+\sigma^{2} / 4-r \sigma \cos \theta_{2}$. One obtains, 


$$
\Delta=\frac{\pi g_{H S}(\eta)}{\sigma^{2}} \int_{D}^{+\infty} r d r \int_{(r-\sigma / 2)^{2}}^{(r+\sigma / 2)^{2}} \frac{d x^{2}}{x} \int_{(x-\sigma / 2)^{2}}^{(x+\sigma / 2)^{2}} f\left(z^{2} / \xi^{2}\right) d z^{2}
$$

where $f\left(z^{2} / \xi^{2}\right)=\exp \left[-\beta V_{G}(z)\right]-1$.

3 Change the order of integration in (11), introducing a cut-off $z<\sigma$ (consistent with the procedure adopted in the simulations to define a bond) and distinguish the cases $D \geq \sigma$ and $D<\sigma$. After a simple but long calculation, $\Delta$ may be expressed as a sum of simple integrals. For $D \geq \sigma$,

$\Delta=4 \pi \sigma^{3} g_{H S}(\eta) \alpha^{2} \int_{-\frac{\delta}{\alpha}}^{\frac{1}{\alpha}} y f(y) F_{1}(y, \alpha, \delta) d y$,

where $\alpha=\xi / \sigma, \delta=(\sigma-D) / \sigma$, and

$F_{1}(y, \alpha, \delta)=\frac{\alpha^{3} y^{3}}{6}+\frac{\alpha^{2} y^{2}}{2}+\alpha y \delta\left(1-\frac{\delta}{2}\right)+\delta^{2}\left(\frac{1}{2}-\frac{\delta}{3}\right)$.
For $D<\sigma$,

$$
\Delta=4 \pi \sigma^{3} g_{H S}(\eta) \alpha^{2}\left(\Delta_{1}+\Delta_{2}+\Delta_{3}\right),
$$

where,

$$
\Delta_{1}=\int_{0}^{\frac{1}{\alpha}} y f(y) F_{1}(y, \alpha, 0) d y
$$

$$
\Delta_{2}=\frac{\alpha \delta(2-\delta)}{2} \int_{0}^{\frac{1-\delta}{\alpha}} y^{2} f(y) d y+\int_{\frac{1-\delta}{\alpha}}^{\frac{1}{\alpha}} y f(y) F_{2}(y, \alpha, \delta) d y
$$

with,

$$
F_{2}(y, \alpha, \delta)=\frac{\alpha y}{2}-\frac{\alpha^{3} y^{3}}{6}-\frac{(1-\delta)^{3}}{3},
$$

and,

$$
\Delta_{3}=\frac{\alpha}{2} \int_{0}^{\frac{\delta}{\alpha}} y^{2} f(y) F_{3}(y, \alpha, \delta) d y+\frac{\delta^{2}}{2}\left(1-\frac{2 \delta}{3}\right) \int_{\frac{\delta}{\alpha}}^{\frac{1-\delta}{\alpha}} y f(y) d y+\int_{\frac{1-\delta}{\alpha}}^{\frac{1}{\alpha}} y f(y) F_{4}(y, \alpha, \delta) d y,
$$

with,

$$
F_{3}(y, \alpha, \delta)=1-\alpha y-(1-\delta)^{2},
$$

and,

$$
F_{4}(y, \alpha, \delta)=\frac{1}{6}\left(1-\alpha^{3} y^{3}\right)+\frac{1}{2}(1-\alpha y)(1-\delta)^{2} .
$$

The percolation line in the $\rho, T$ diagram (see the main text) is obtained by setting $p_{b}=\frac{1}{n-1}=\frac{1}{2}$ in Eq. (9). The coexistence line is calculated from the equality of the pressures and chemical potentials obtained from $\beta f$. The bonding fraction $p_{b}$ is obtained by solving Eq. (9), in single phase thermodynamic states. For state points in the coexistence region $\left(k_{B} T / \epsilon<0.0678\right.$ in Fig. 1 at $\rho=0.2) p_{b}$ is calculated using the so-called lever rule: $p_{b}(\rho, T)=p_{b, l}+x\left(p_{b, v}-p_{b, l}\right)$, with $x=\left(\rho_{l}-\rho\right) /\left(\rho_{l}-\right.$ $\rho_{v}$ ), where $\rho_{v}$ and $\rho_{l}$ are the coexistence gas and liquid densities at $T$, and $p_{b, v}$ and $p_{b, l}$ are the bonding fractions in the coexisting gas and liquid phases at $T$.

Acknowledgments. We acknowledge fruitful discussions with Emanuela Del Gado and financial support from the Portuguese Foundation for Science and Technology (FCT) under Contracts nos. EXCL/FIS-NAN/0083/2012, UID/FIS/00618/2013, and $\mathrm{IF} / 00255 / 2013$.

* csdias@fc.ul.pt

$\dagger$ jmtavares@fc.ul.pt

‡ nmaraujo@fc.ul.pt

$\S$ mmgama@fc.ul.pt

[1] J. Dobnikar, A. Snezhko, and A. Yethiraj, "Emergent colloidal dynamics in electromagnetic fields," Soft Matt. 9, 3693 (2013).

[2] E. Zaccarelli, "Colloidal gels: equilibrium and nonequilibrium routes," J. Phys.: Condens. Matter 19, 323101 (2007).

[3] S. L. Elliott, R. J. Butera, L. H. Hanus, and N. J. Wagner, "Fundamentals of aggregation in concentrated dispersions: fiber-optic quasielastic light scattering and linear viscoelastic measurements." Faraday discuss. 123, 369 (2003).

[4] B. Ruzicka, E. Zaccarelli, L. Zulian, R. Angelini, M. Sztucki, A. Moussaïd, T. Narayanan, and F. Sciortino, "Observation of empty liquids and equilibrium gels in a colloidal clay," Nat. Mater. 10, 56 (2011).

[5] F. Sciortino, S. Mossa, E. Zaccarelli, and P. Tartaglia, "Equilibrium cluster phases and low-density arrested disordered states: The role of short-range attraction and long-range repulsion," Phys. Rev. Lett. 93, 055701 (2004).

[6] D. Chakrabarti, H. Kusumaatmaja, V. Rühle, and D. J. 
Wales, "Exploring energy landscapes: from molecular to mesoscopic systems." Phys. Chem. Chem. Phys. 16, 5014 (2014).

[7] E. Zaccarelli, I. Saika-Voivod, S. V. Buldyrev, A. J. Moreno, P. Tartaglia, and F. Sciortino, "Gel to glass transition in simulation of a valence-limited colloidal system," J. Chem. Phys. 124, 124908 (2006).

[8] J. Zhang, E. Luijten, and S. Granick, "Toward design rules of directional Janus colloidal assembly," Ann. Rev. Phys. Chem. 66, 581 (2015).

[9] J. A. Paulson, A. Mesbah, X. Zhu, M. C. Molaro, and R. D. Braatz, "Control of self-assembly in micro- and nano-scale systems," J. Proc. Cont. 27, 38 (2015).

[10] P. J. Lu and D. A. Weitz, "Colloidal particles: Crystals, glasses, and gels," Annu. Rev. Condens. Matter Phys. 4, 217 (2013).

[11] E. Duguet, A. Désert, A. Perro, and S. Ravaine, "Design and elaboration of colloidal molecules: an overview." Chem. Soc. Rev. 40, 941 (2011).

[12] J. Hu, S. Zhou, Y. Sun, X. Fang, and L. Wu, "Fabrication, properties and applications of Janus particles," Chem. Soc. Rev. 41, 4356 (2012).

[13] I. Kretzschmar and J. H. Song, "Surface-anisotropic spherical colloids in geometric and field confinement," Curr. Op. Coll. Interf. Sci. 16, 84 (2011).

[14] S. Sacanna and D. J. Pine, "Shape-anisotropic colloids: Building blocks for complex assemblies," Curr. Op. Coll. Interf. Sci. 16, 96 (2011).

[15] M. J. Solomon, "Directions for targeted self-assembly of anisotropic colloids from statistical thermodynamics," Curr. Op. Coll. Interf. Sci. 16, 158 (2011).

[16] A. B. Pawar and I. Kretzschmar, "Fabrication, assembly, and application of patchy particles," Macromol. Rapid Commun. 31, 150 (2010).

[17] S. Sacanna, D. J. Pine, and G.-R. Yi, "Engineering shape: the novel geometries of colloidal self-assembly," Soft Matt. 9, 8096 (2013).

[18] V. N. Manoharan, "Colloidal matter: Packing, geometry, and entropy," Science 349, 1253751 (2015).

[19] E. Zaccarelli, S. V. Buldyrev, E. La Nave, A. J. Moreno, I. Saika-Voivod, F. Sciortino, and P. Tartaglia, "Model for reversible colloidal gelation," Phys. Rev. Lett. 94, 218301 (2005).

[20] N. A. Mahynski and A. Z. Panagiotopoulos, "Grafted nanoparticles as soft patchy colloids: Self-assembly versus phase separation," J. Chem. Phys. 142, 074901 (2015).

[21] E. G. Noya and E. Bianchi, "Phase behaviour of inverse patchy colloids: effect of the model parameters," J. Phys.: Condens. Matter 27, 234103 (2015).

[22] F. Romano, J. Russo, and H. Tanaka, "Influence of Patch-Size Variability on the Crystallization of Tetrahedral Patchy Particles," Phys. Rev. Lett. 113, 138303 (2014).

[23] J. Russo, P. Tartaglia, and F. Sciortino, "Reversible gels of patchy particles: Role of the valence," J. Chem. Phys. 131, 014504 (2009).

[24] E. Bianchi, J. Largo, P. Tartaglia, E. Zaccarelli, and F. Sciortino, "Phase diagram of patchy colloids: Towards empty liquids," Phys. Rev. Lett. 97, 168301 (2006).

[25] E. Bianchi, R. Blaak, and C. N. Likos, "Patchy colloids: state of the art and perspectives," Phys. Chem. Chem. Phys. 13, 6397 (2011).

[26] K. Kroy, M. E. Cates, and W. C. K. Poon, "Cluster mode-coupling approach to weak gelation in attractive colloids," Phys. Rev. Lett. 92, 148302 (2004).

[27] S. Manley, H. M. Wyss, K. Miyazaki, J. C. Conrad, V. Trappe, L. J. Kaufman, D. R. Reichman, and D. A. Weitz, "Glasslike arrest in spinodal decomposition as a route to colloidal gelation," Phys. Rev. Lett. 95, 238302 (2005).

[28] C. S. Dias, N. A. M. Araújo, and M. M. Telo da Gama, "Mixtures of functionalized colloids on substrates," J. Chem. Phys. 139, 154903 (2013).

[29] F. Sciortino, C. De Michele, S. Corezzi, J. Russo, E. Zaccarelli, and P. Tartaglia, "A parameter-free description of the kinetics of formation of loop-less branched structures and gels," Soft Matt. 5, 2571 (2009).

[30] A. J. Bray, "Coarsening dynamics of phase-separating systems," Phil. Trans. R. Soc. Lond A 361, 781 (2003).

[31] B. Ruzicka and E. Zaccarelli, "A fresh look at the Laponite phase diagram," Soft Matt. 7, 1268 (2011).

[32] N. R. Bernardino and M. M. Telo da Gama, "Reentrant wetting of network fluids," Phys. Rev. Lett. 109, 116103 (2012).

[33] X. Xu, J. Wang, J. P. Lv, and Y. Deng, "Simultaneous analysis of three-dimensional percolation models," Front. Phys. 9, 113 (2014).

[34] D. Stauffer and A. Aharony, Introduction to percolation theory (Taylor \& Francis, London, 1994).

[35] J. Midya and S. K. Das, "Kinetics of Vapor-Solid Phase Transitions: Structure, Growth, and Mechanism," Phys. Rev. Lett. 118, 165701 (2017).

[36] H. Schmidle, S. Jäger, C. K. Hall, O. D. Velev, and S. H. L. Klapp, "Two-dimensional colloidal networks induced by a uni-axial external field," Soft Matt. 9, 2518 (2013).

[37] S. H. L. Klapp, "Collective dynamics of dipolar and multipolar colloids: from passive to active systems," Curr. Op. Coll. Interf. Sci. 21, 76 (2016).

[38] D. J. Kraft, J. Hilhorst, M. A. P. Heinen, M. J. Hoogenraad, B. Luigjes, and W. K. Kegel, "Patchy polymer colloids with tunable anisotropy dimensions," J. Phys. Chem. B 115, 7175 (2011).

[39] M. Sabapathy, S. D. Christdoss Pushpam, M. G. Basavaraj, and E. Mani, "Synthesis of Single and Multipatch Particles by Dip-Coating Method and SelfAssembly Thereof," Langmuir 31, 1255 (2015).

[40] N. Kern and D. Frenkel, "Fluid-fluid coexistence in colloidal systems with short-ranged strongly directional attraction," J. Chem. Phys. 118, 9882 (2003).

[41] C. S. Dias, N. A. M. Araújo, and M. M. Telo da Gama, "Effect of the number of patches on the growth of networks of patchy colloids on substrates," Mol. Phys. 113, 1069 (2015).

[42] N. Khalil, A. de Candia, A. Fierro, M. P. Ciamarra, and A. Coniglio, "Dynamical arrest: interplay of glass and gel transitions." Soft Matt. 10, 4800 (2014).

[43] C. S. Dias, C. Braga, N. A. M. Araújo, and M. M. Telo da Gama, "Relaxation dynamics of functionalized colloids on attractive substrates," Soft Matt. 12, 1550 (2016).

[44] O. A. Vasilyev, B. A. Klumov, and A. V. Tkachenko, "Precursors of order in aggregates of patchy particles," Phys. Rev. E 88, 012302 (2013).

[45] S. Plimpton, "Fast parallel algorithms for short-range Molecular Dynamics," J. Comp. Phys. 117, 1 (1995).

[46] M. G. Mazza, N. Giovambattista, H. E. Stanley, and F. W. Starr, "Connection of translational and rotational 
dynamical heterogeneities with the breakdown of the Stokes-Einstein and Stokes-Einstein-Debye relations in water," Phys. Rev. E 76, 031203 (2007).

[47] F. Sciortino, E. Bianchi, J. F. Douglas, and P. Tartaglia, "Self-assembly of patchy particles into polymer chains: A parameter-free comparison between Wertheim theory and Monte Carlo simulation," J. Chem. Phys. 126, 194903 (2007).

[48] J. A. Barker and D. Henderson, "Perturbation Theory and Equation of State for Fluids. II. A Successful Theory of Liquids," J. Chem. Phys. 47, 4714 (1967).

[49] M. S. Wertheim, "Fluids of dimerizing hard spheres, and fluid mixtures of hard spheres and dispheres," J. Chem. Phys. 85, 2929 (1986). 


\title{
Supplementary Information: Relaxation dynamics of a network fluid inside the liquid-gas coexistence region
}

\author{
C. S. Dias, ${ }^{1,2, *}$ J. M. Tavares,${ }^{2,3, \dagger}$ N. A. M. Araújo, ${ }^{1,2, \ddagger}$ and M. M. Telo da Gama ${ }^{1,2, \S}$ \\ ${ }^{1}$ Departamento de Física, Faculdade de Ciências, \\ Universidade de Lisboa, 1749-016 Lisboa, Portugal \\ ${ }^{2}$ Centro de Física Teórica e Computacional, Universidade de Lisboa, 1749-016 Lisboa, Portugal \\ ${ }^{3}$ Instituto Superior de Engenharia de Lisboa, ISEL, \\ Avenida Conselheiro Emídio Navarro, 1 1950-062 Lisboa - Portugal
}

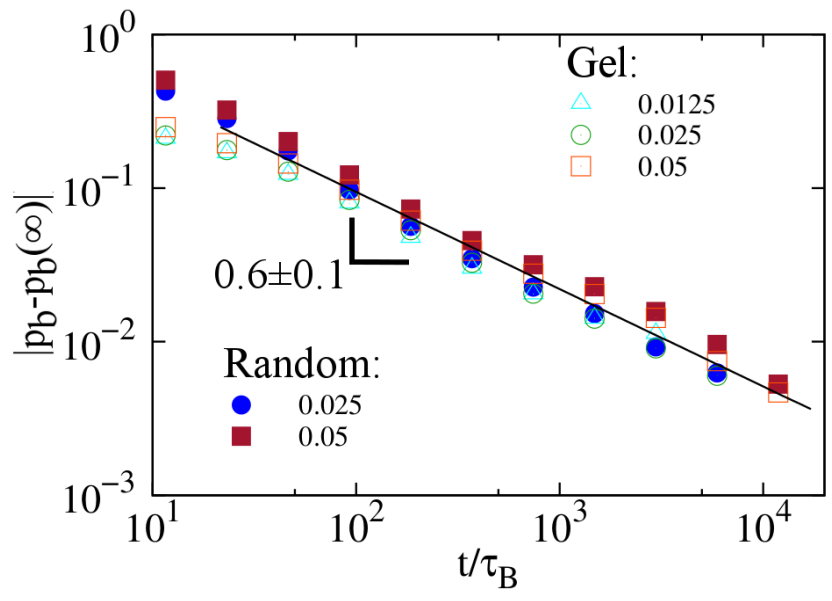

FIG. S1. Convergence of the bonding fraction to the asymptotic value, $\left|p_{b}-p_{b}(\infty)\right|$, at temperatures $k_{B} T / \varepsilon=$ $\{0.0125,0.025,0.05\}$ starting from a singly connected tree-like gel at temperatures $k_{B} T / \varepsilon=\{0.025,0.05\}$ and starting from a fully disconnected suspension, where the time is in units of the Brownian time $\tau_{B}=\sigma^{2} / D_{t}, \sigma$ is the particle diameter and $D_{t}$ the diffusion coefficient. Numerical results were obtained by averaging over 10 samples in a box of length $L=16$, in units of particle diameter.

\section{BONDING FRACTION}

Figure S1 reveals that the relaxation proceeds as a power law in time, with exponent $0.6 \pm 0.1$, which is observed for different initial conditions and temperatures below $k_{B} T / \varepsilon=0.075$.

In Fig. $\mathrm{S} 2$ we plot the asymptotic values of $p_{b}$ for different system sizes showing that size effects are not sig- nificant.

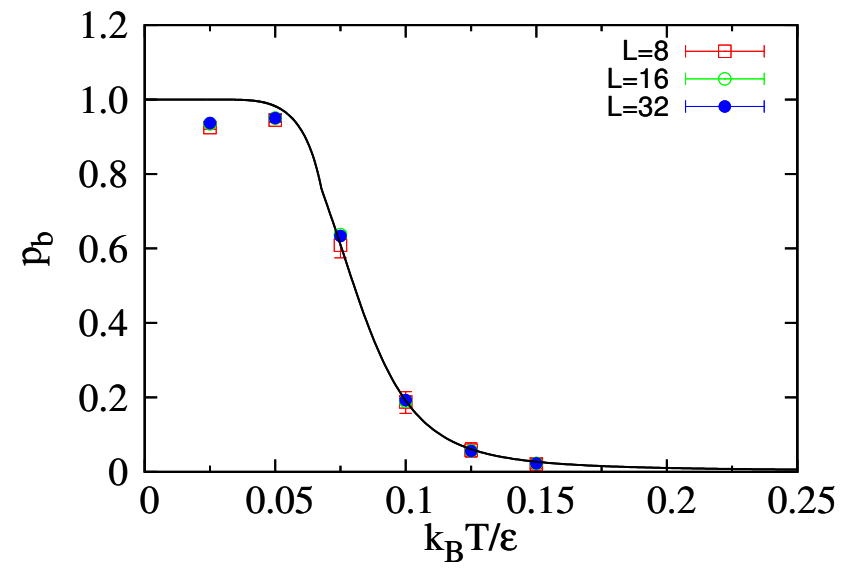

FIG. S2. Bonding fraction $p_{b}$ as a function of the rescaled temperature $k_{B} T / \varepsilon$, obtained from the asymptotic extrapolation of the numerical data for systems of linear size $L=$ $\{8,16,32\}$. The solid line is the equilibrium value predicted by Wertheim's first-order perturbation theory. Numerical results were obtained by averaging over 10 samples.

\section{RELAXATION DYNAMICS}

In Fig. S3, we plot the average size of clusters as a function of time for all particles and for particles with three bond (assumed to be in the liquid phase). We find that a cluster with all the particles is formed on a timescale much shorter than that corresponding to the scaling regime reported above.

\footnotetext{
* csdias@fc.ul.pt

$\dagger$ jmtavares@fc.ul.pt
}

$\S$ mmgama@fc.ul.pt 


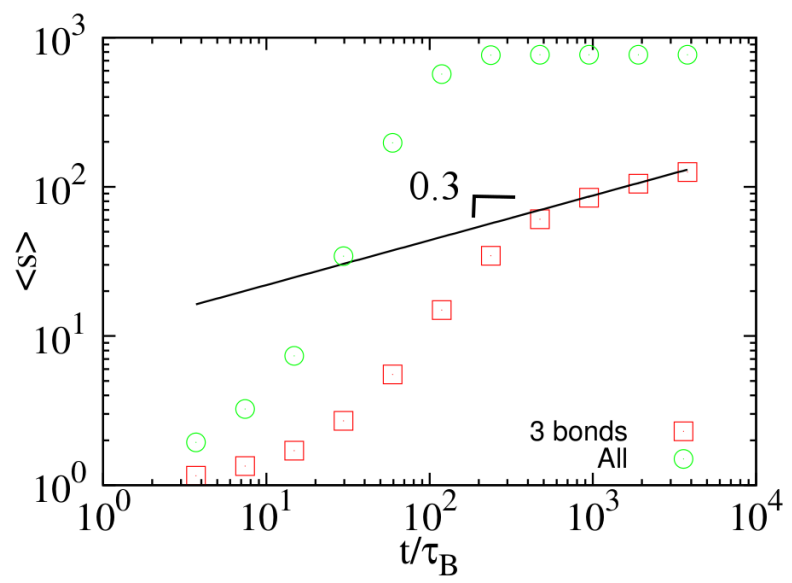

FIG. S3. Average size of clusters $\langle s\rangle$ of particles (open circles) and of fully bonded particles (open squares) as a function of time. Numerical results obtained by averaging over 10 samples of a system of size $L=16$. 